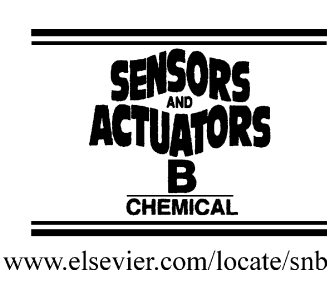

\title{
Continuous sampling and analysis by on-chip liquid/solid chromatography
}

\author{
Mario Schlund $^{\mathrm{a}, *}$, Scott E. Gilbert ${ }^{\mathrm{b}}$, Silvan Schnydrig ${ }^{\mathrm{a}}$, Philippe Renaud ${ }^{\mathrm{a}}$ \\ a Microsystems Laboratory, STI-LMIS, EPFL, CH-1015 Lausanne, Switzerland \\ ${ }^{\mathrm{b}}$ Crystal Vision Microsystems LLC, Edmonds, WA 98020, United States
}

Received 18 August 2006; received in revised form 3 October 2006; accepted 10 October 2006

\begin{abstract}
This paper describes a lab-on-a-chip device for continuous liquid/solid chromatography measurements.

Chromatographic separations of phenolic test solutions as well as of vitamins are illustrating the ability and versatility of the system. The dependence of the peak height and width with respect to the injected plug and the saturation limits of the microchip column have been investigated and good correlation to the theoretical predictions have been observed.

The focus of the system design is on simplification of both fabrication complexity and application. To this end, large bore flow-through fluid introduction channels have been integrated onto the chip to allow aliquoting from meso-scale flows of sample (up to tens of milliliters per minute). Repeatable plug injections and reproducible chromatographic separations were achieved in an open-tubular on-chip microcolumn with $\mathrm{C}_{8}$ stationary phase coating. The entire system allows for a rapid cycle time for multiple analyses, exchange of analyte and mobile phase in less than a minute, and complete cleaning cycles within a few seconds only. This makes it a suitable candidate for on-line process analysis applications.
\end{abstract}

(C) 2006 Elsevier B.V. All rights reserved.

Keywords: On-chip chromatography; On-line analysis; Continuous sampling; Microfluidics

\section{Introduction}

Microfabricated planar separation devices have been extensively studied for the past 15 years and touted for their size, speed and low cost compared to conventional instrumentation. The literature contains hundreds of articles describing electrophoretic and electrokinetically driven devices, such as capillary electrophoresis (CE), reversed-phase capillary electrochromatography (CEC) and micellular electrokinetic chromatography (MEKC) [1-5]. These devices utilize the principle of electroosmotic flow for fluid propulsion, which is more amenable to miniaturization on a planar chip format. In addition, electrophoretic techniques find abundant application in biomolecular analysis, a tremendous market driver at the present time. On the other hand, chip-based pressure-driven liquid chromatographic (LC) systems have been slow to find extensive interest, and have been pioneered by a handful of workers [6-20]. This is mainly due to lack of ease of flow control as is the case with electrokinetically driven devices where mechanical pumps and

\footnotetext{
* Corresponding author. Tel.: +41 2169366 06; fax: +41 216935950.

E-mail address: mario.schlund@epfl.ch (M. Schlund).
}

valves are obviated, and to the difficulty in integration of on-chip injectors and mechanical valves [21]. Despite these handicaps, there is much motivation to develop microfabricated LC systems, as LC is still the workhorse analytical method in most laboratories for small molecule analysis. Pressure-driven capillary LC systems in the form of microbore and nanobore HPLC columns have proven their superiority to conventional HPLC columns in terms of resolution, speed and solvent and sample conservation, as well as their ability to be coupled to mass spectrometers for proteomics analysis and as a high-throughput analytical tool for combinatorial drug and metabolome screening. There is a growing need for miniaturizing LC by combining a capillary column with injectors and detectors small enough to take advantage of the ultra-small column volume presented by the nanobore capillary. This is best done by microfabrication, using clever design of a microfluidic network to combine a robust injection system with capillary column, and a detector based on integrated electrodes, diffraction grating or a total internal reflectance surface in a fully integrated planar package.

Although most of the motivation for miniaturization of pressure-driven LC comes from the need to fulfill niches in the biotechnology arena, an application that has been overwhelmingly overlooked is that of on-line analysis in chemical and 
pharmaceutical production. Conventional HPLC has not been widely employed as an on-line analytical tool because of slowness, size, lack of robustness and sampling complexities and running expense. Microfabricated LC offers many solutions that address these issues and lower the barrier to implementation of the technique. We have focused our efforts on solutions for representative sampling and reproducible injection. Additionally, an open tubular approach to the separation column, as opposed to a packed column approach, has been adopted to capitalize on the superior performance of the former over the latter that are possible at low pressures if the column dimensions are optimized. Practically speaking, elimination of a mechanical high-pressure pump is a great advantage for an on-line or portable monitoring device.

This paper addresses two main issues that have impeded the implementation of chip-based LC for on-line analysis. The first is an adequate injection technique that is fully integrated and reliable, not requiring a syringe pump to load the chip. The second is a robust sampling method from a macroscopic flow. In reference to the first point, on-chip injection methods in pressure-driven systems that have been reported in the literature have used a double-T junction type microfluidic design that emulates an HPLC injector loop. In this design, an injection plug of fixed volume is contained in the main channel segment between the two side branches. The design requires use of syringe pumps or a standard HPLC injection valve $[6,12,15]$. However, it is difficult to assure repeatability using this technique, as the confining segment is easily overflowed, and the extremely low flow rates for sample loading are difficult to obtain. An improvement in terms of injection plug control is the so-called pressure-pinched injection, where the continuous analyte flow is compressed at the injection cross from either side by side streams of mobile phase, in order to minimize the volume while at the same time obtaining reproducible injection plugs [22,23]. This design relies on very delicate balancing of flow rates to work properly. A variation on this theme was devised by Vahey et al. [24], where a pressure-pinched injection scheme was implemented, but allowing variable plug length to be injected into the separation channel. Again, a delicate flow rate balance must be maintained. Another interesting method is the so-called pressure-pulse injection developed for electrophoretic microchips [25]. In this paper, we will introduce a very simple but nonetheless robust injection method also based on flowing analyte and mobile phase streams, but using a gated injection approach whereby the confluence of two laminar flows at a injection cross can be made to exclude the analyte from entering the separation column by the mobile phase stream, which exclusively flows in this channel, until an intentional perturbation in the flow rate of the latter allows the former to enter. This technique has been employed in plug formation on electroosmotically driven microfluidic chips, where voltage switching is used to gate the injection, and is a well-known injection method for chip-based electrophoretic separations [26-28]. In this work, perturbation of driving pressures is used to create repeatable injections with injection volumes dependent on the duration of the pressure change and the flow velocity.

The second point to be addressed is representative sampling. Rapid renewal and/or purging of sample inlet lines to the chip is of paramount importance for obtaining representative samples in on-line analysis of microreactors and macroscale reactors, and to eliminate cross-talk between samples. In order to accomplish this, it is necessary to design the system so that meso-scale flow rates (tens of microliters to milliliters per minute) of fresh sample liquid can pass by the inlet leading to the on-chip injector, where fresh aliquots can be drawn from the stream. To this end, a flowthrough strategy for sample and mobile phase introduction was conceived, permitting continuous or intermittent flow of fresh sample stream on board the chip, with injection of an aliquot of the sample stream executed in a periodic or random fashion. Large flow-through channels $(200 \mu \mathrm{m} \times 1 \mathrm{~mm} \times 5 \mathrm{~mm})$ permit sample and mobile phase streams to flow either separately or simultaneously on the chip. This sampling approach has found precedent in microfabricated electrophoretic devices [29-33], but has not been attempted for chip-based LC.

\section{Experimental}

\subsection{Chip microfabrication and interconnection}

The microfabrication of the float-glass wafers is based on a four-mask process (Fig. 1). Separation columns and bypass channels in the bottom wafer are HF-etched through an AZ 5214 photoresist mask (Fig. 1(a)), while the recessed Ti/Pt electrodes are defined by a lift-off process using the same photoresist (Fig. 1(b)). The cover plate consisted of a float-glass wafer, which contains the powder-blasted flow-through channels and fluid access ports. Powder blasting was performed manually using $60 \mu \mathrm{m}$ corundum grit. For precise positioning of holes and other structures, the blasting pattern was pre-defined photolithographically using a laminated dry resist specially designed for decorative sandblasting purposes (RapidMask ${ }^{\mathrm{TM}}$, Photoabrasive Systems, Duluth, MN, USA), and exposing to uncollimated UV light through a transparency mask printed with a high-dpi inkjet printer.

Top and bottom glass wafers were anodically bonded [34,35] using an RF-sputtered $40 \mathrm{~nm}$ thick amorphous Si layer as barrier for wafer-to-wafer sodium ion transfer. The bonding is achieved by applying $700 \mathrm{~V}$ at a temperature of $360^{\circ} \mathrm{C}$ during $30 \mathrm{~min}$ in a muffle furnace under an ambient atmosphere. Finally, the wafers were diced into single chips. Electrode access pads were liberated by half-through dicing as shown in Fig. 1(d). Key channel dimensions and materials parameters are presented in Table 1, with a photograph of the chip shown in Fig. 2(a).

Table 1

Chromatography microchip characteristics

\begin{tabular}{ll}
\hline Channel width $(\mu \mathrm{m})$ & 50 \\
Channel height $(\mu \mathrm{m})$ & 7 \\
Flow-through channel height $(\mathrm{mm})$ & 0.2 \\
Separation channel length (injection cross to detector) $(\mathrm{cm})$ & 11.1 \\
Separation channel length (injection cross to outlet) $(\mathrm{cm})$ & 11.6 \\
Bypass channel length $(\mathrm{cm})$ & 5.4 \\
Wafer material & Float glass \\
Electrode material & Ti/Pt \\
\hline
\end{tabular}


Microfluidic channels:

(a)

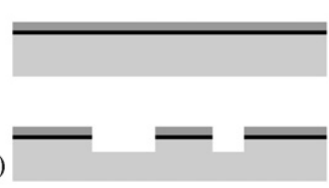

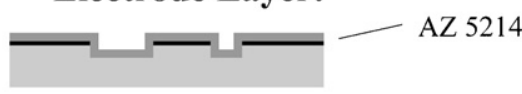

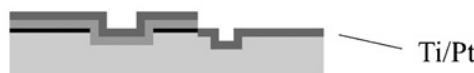

(b)

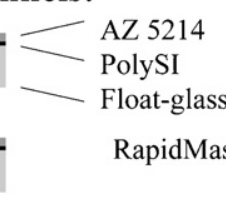

(c)

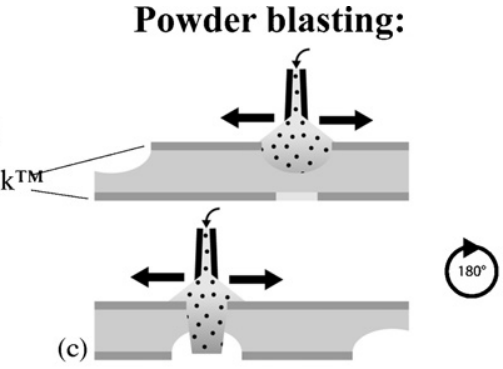

Assembly by anodic bonding and dicing:

(d)

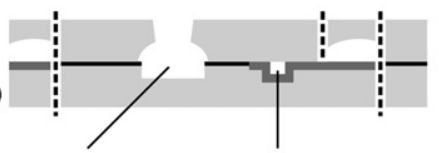

Flow-through channel Sepration column with with in- and outlets detection electrodes

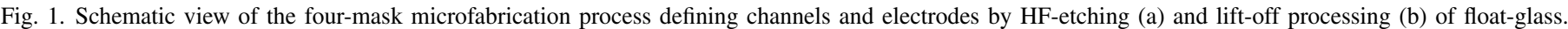

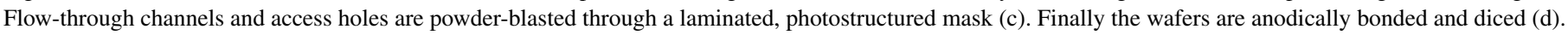
Detection electrodes access pads are liberated by partial dicing.

The column walls were then coated using a sol-gel technique. This procedure yields a thin coating of a silica hybrid gel on the column wall which contains the functional layer. In our case this is a mixture of tetraethoxysilane/tetraethoxysilane- $\mathrm{C}_{8}$ that yields a $\mathrm{C}_{8}$ layer acting as stationary phase, follow- ing the preparation shown by Constantin et al. [36,37]. The sol-gel precursor solution is pumped into the column, allowed to stay for several minutes and then forced out of the column by nitrogen overpressure before the chip is dried overnight under nitrogen flow at $120^{\circ} \mathrm{C}$. This process yields

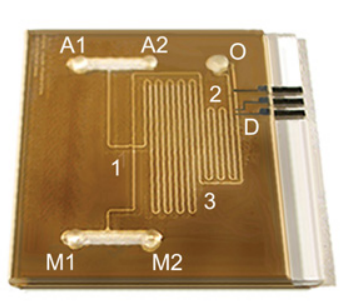

\section{$\mathrm{A}_{1 / 2} \quad$ analyte flow-trough inlet/outlet}

$\mathrm{M}_{1 / 2}$ mobile phase flow-trough inlet/outlet

D detection electrodes

O outlet port

1 injection cross

2 bypass channel

3 separation column

$V_{1} \ldots 4$ valves

P1 applied pressure
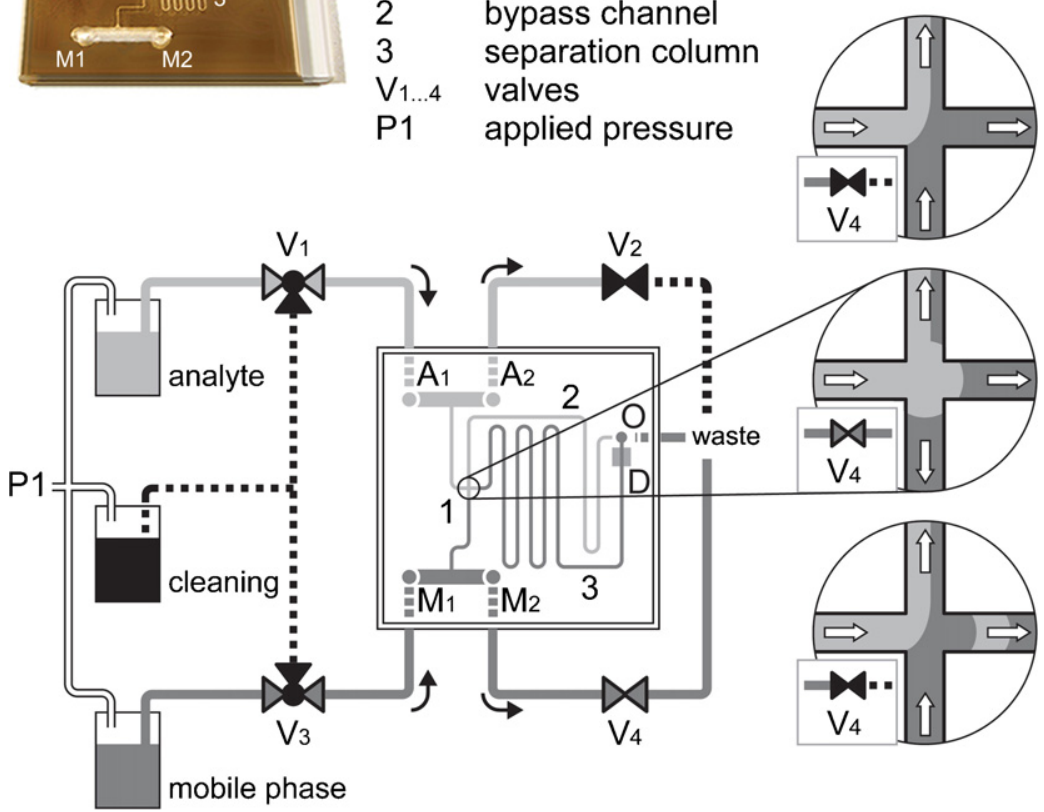

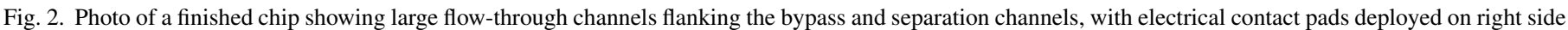

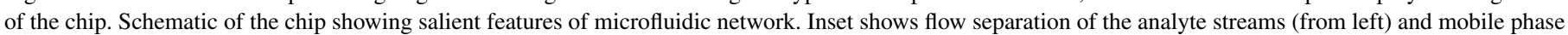

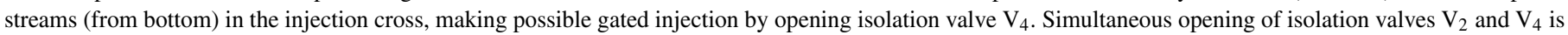
used for the optional washing cycles.

Please cite this article in press as: M. Schlund et al., Continuous sampling and analysis by on-chip liquid/solid chromatography, Sens. Actuators B: Chem. (2006), doi:10.1016/j.snb.2006.10.021 
a reversed-phase open-tubular column with high-surface area.

\subsection{Chromatographic microchannel design}

The overall dimensions of the separation channel were calculated in accordance with theory for rectangular open channel reversed phase liquid chromatography as presented by Giddings et al. [38]. We updated Giddings' theory with modified Golay coefficients as suggested by Poppe [39], to account for the strong side-wall induced dispersion in high-aspect ratio rectangular channels. Using this theoretical framework, the separation channels were designed to perform optimally at flow rates of up to $100 \mathrm{nl} / \mathrm{min}$, with the channel dimensions listed in Table 1 . These parameters allow for operation at relatively low pressures of less than 1 bar, thus pressure-driven flow could be obtained without resorting to the use of a high-pressure pump. The pressuredriven system will be described in detail in the next section. As the separation channel was $11 \mathrm{~cm}$ long, it was necessary to etch it in a serpentine folded configuration to fit the channel on the $20 \mathrm{~mm} \times 20 \mathrm{~mm}$ chip format. Attention was paid to the radii of curvature of the channel turns and length of straight channel segments, so as to minimize contributions to band dispersion induced by turns in the folded microchannel (distortion of the band due to the racetrack effect) over contributions from axial diffusion alone. Equations developed by Griffiths and Nilson $[40,41]$, minimum radii of curvature were calculated with respect to straight segment lengths, which need to be sufficiently long to allow time for transverse diffusion to erase the distortion incurred after each turn by Taylor-Aris dispersion. The calculations are based on the Péclet number for the channel, taking into account the channel width, anticipated fluid velocities and solute diffusion coefficients.

\subsection{Chip-fluid interface}

A single piece chip interface was designed for easy mounting and dismounting of the chips. The interface was machined from PEEK block, with flat bottom ports drilled and tapped on the side of the mount for efficient attachment of fluid delivery lines to the body of the mount. The chip seats on top of the block, from which $1 \mathrm{~mm}$ access holes were drilled to line up with fluid inlet ports on the chip. The access holes intersect orthogonally with similar holes originating from the ports for fluid delivery to the chip. Holes mating with the chip ports are countersunk to accommodate small Kalrez $o$-rings for proper sealing. The chip is pressed to the interface block by a cover piece in PMMA or other transparent plastic that is secured to the mount with M2 screws. Electrical contact to the chip was made via juxtaposition of a $1 \mathrm{~mm}$ contact pitch microconnector (Samtec) soldered on a small printed circuit board for easy mounting on the chip interface block. Wires soldered to the circuit board were connected to the electrochemical detector.

\subsection{Experimental set-up and fluidic control}

A crucial aspect of lab-on-a-chip setups is the interconnection between the chip, electronics and fluidics, which are frequently cumbersome and starting point of a large number of problems related to the lab-on-a-chip technology. Conventional tubing and interconnection techniques often present dead volumes that are orders of magnitude larger than the total volume of the microfluidic channels on the chip itself. Two main strategies tend to minimize those dead volumes. A first approach consists of integrating those parts responsible for dead volumes, as for example, check valves. The inconvenience of a maximized integration is an important raise in fabrication complexity [42].

Because controlled fluid pressures and not flow rate was essential for proper operation of the experimental set-up, we found it more efficient to use pressurized reservoirs for both sample and mobile phase delivery, providing precise pressure control as opposed to pumps. Sample and mobile phase were contained in separate $100 \mathrm{ml}$ glass bottles using fluid distribution caps with threaded flat-bottom ports (Bohlender $\mathrm{GmbH}$ ). The liquid pressure was controlled by varying the pressure in the head-space over the liquids using pressurized air, with control of the gas pressure assured by a manual high-precision pressure regulator and precision gauge over a range of $0-1.5$ bar (Marsh Bellofram). For most experiments, the system was operated between 300 and 1500 mbar, with 500-1000 mbar being typical.

Fluid delivery lines and connectors, Teflon ${ }^{\circledR}$ tubing and ETFE flangeless fittings, respectively, were obtained from Upchurch Scientific (Oak Harbor, WA, USA). Fluid flow was controlled by normally closed solenoid isolation valves (BioChem Valve/Omnifit). The valves were actuated using solid state relays controlled by logic signals emanating from the data acquisition board (PCI-DAS6014 D/A-A/D-DIO board, Measurement Computing).

For data acquisition and system control, a LabVIEW ${ }^{\circledR}$ virtual instrument (VI) manager was written using the appropriate drivers for the data acquisition board as supplied by the manufacturer.

\subsection{Sampling and injection methodologies}

The design principle of the injection system calls for sample and mobile phase inlet channels to intersect orthogonally, with the sample inlet channel continuing as the separation channel after the intersection. Fig. 2 shows a schematic of the device. Large-bore flow-through inlets (A1, M1) are connected to reservoirs with applied pressure $P_{1}$, containing analyte and mobile phase, respectively, while outlet $(\mathrm{O})$ of the microfluidic manifold is connected to atmospheric pressure. Segment 3 is the separation column while segment 2 is the analyte bypass channel. These channels intersect with the flow-through inlets A1 and M1, and access fluids flowing through them. The liquids are driven by the resulting pressure drop from the point of intersection with $\mathrm{A}$ and $\mathrm{M}$ to the outlet $\mathrm{O}$. A higher flow resistance in the separation column compared to that of the bypass channel causes the analyte flow to divert into the bypass channel, while the mobile phase is forced to flow into the separation channel due to the inability of streamlines to cross in laminar flow. Mobile phase can also flow into the bypass channel, and the ratio of analyte to mobile phase flowing in this branch is controlled by the 
fluid pressures. A continuous flow of part of the mobile phase into the bypass channel screens the separation column from the analyte, thus preventing analyte from flowing unintentionally into the separation column, either by diffusion into the mobile phase at the injection cross or by small pressure variations at the inlets (Fig. 2, inset). The method is analogous to gated injection schemes used in earlier electrophoresis microchips by Ramsey and co-workers [27] and Harrison et al. [43]. Pressure variations in either stream will result in a change in the flow rate ratio. To engender plug injection into the separation channel, a reduction of the mobile phase flow rate by intentional momentary release of the pressure, or by constriction of the flow, will allow analyte stream to begin flowing into the separation channel. Conversely, a higher overpressure in the analyte stream can cause the mobile phase to recede and overtake its flow down the separation channel. The duration of the pressure perturbations is easily controlled using the external valves. We have used two methods to accomplish this; one, releasing gas pressure in the headspace over the mobile phase through a leak valve, and two, opening of an isolation valve $\left(\mathrm{V}_{4}\right)$ connected to the flow-through tubing downstream of the chip, releasing hydrostatic pressure in the line.

All results shown in this paper have been obtained with the latter method, shown in the scheme of Fig. 2. The same isolation valves $\left(\mathrm{V}_{2}\right.$ and $\left.\mathrm{V}_{4}\right)$ are simultaneously used for the optional washing cycles. If one wishes to change analytes or mobile phases, the pressurized reservoirs are simply disconnected and exchanged via the manual switching valves $\left(V_{1}\right.$ and $\left.V_{3}\right)$. When the new reservoirs are connected, a washing cycle can be carried out. This takes from a few seconds (only analyte change) up to a few minutes (analyte and mobile phase), depending on the chosen flow speed.

\subsection{Electrochemical detection}

As electrochemical detection is a surface sensitive technique rather than being volume sensitive as is optical detection, it is strictly dependent on the analyte concentration rather than on the number of molecules present in the detector volume. Thus, the detection volume can be made arbitrarily small.

DC amperometric electrochemical detection was accomplished using a BioAnalytical Systems 4B-LC HPLC electrochemical detector, with the output signal conditioned using the unit's internal analogue low pass filter set at a $0.3 \mathrm{~Hz}$ cut-off to remove mostly $50 \mathrm{~Hz}$ noise. The output signal represented the electrochemical current as a function of time and peak heights in the chromatographic data are given in units of nanoamperes. Platinum working, pseudo-reference and counter electrodes were deposited directly in the separation channel near the exit, with the working electrode upstream of the others. These were $100 \mu \mathrm{m}$ wide and spaced apart by $1 \mathrm{~mm}$. The detection volume can be taken as the space immediately surrounding the working electrode, and consists of a volume of approximately $35 \mathrm{pl}$, or $0.1 \%$ of the total column volume of $35 \mathrm{nl}$. The working electrode potential was set at $+1.0 \mathrm{~V}$ versus the Pt pseudo-reference. As the unit had no pulsed detection capability, the applied potential was inverted after each separation for five subsequent periods of $5 \mathrm{~s}$.

\subsection{Chemicals}

All chemicals used in this work were from Acros Organics and Aldrich as received, and solutions were made up in ultrapure water and HPLC-grade organic solvents.

\section{Results and discussion}

\subsection{Microfluidic behavior}

The entire system turned out to be very robust and simple to handle. Flow injection tests were carried out a priori with two liquids containing different food dyes in order to visualize the functionality of the design. For quantization of injection repeatability, optical measurements of plug injection reproducibility as a function of injection time and applied pressure (or mean flow velocity) have been measured using a CCD camera and fluorescent analyte (saturated fluorescein solution at $\mathrm{pH}$ 9.0). Visual observation is shown in the sequence of fluorescence micrographs presented in Fig. 3.

The integration of the fluorescence intensity determined the quantity of the injected analyte. In order to have sufficient analyte intensity, fluorescence tests were carried out using chips with a column of $10 \mu \mathrm{m}$ depth and $11 \mathrm{~cm}$ length. These measurements showed a relatively good repeatability and a linear behavior of the plug volume versus injection time. Nevertheless, as one camera pixel corresponds to $4.1 \mu \mathrm{m}^{2}$, this is a major source of error.
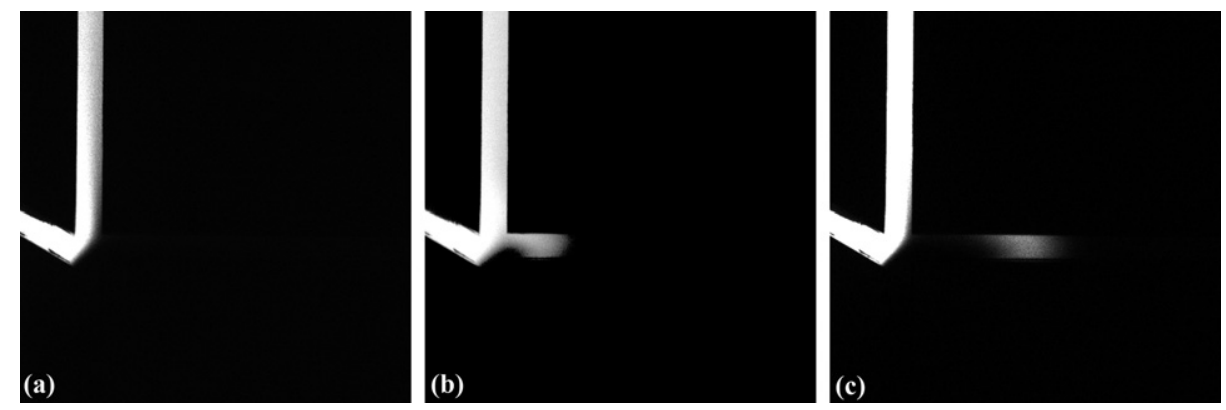

Fig. 3. Fluorescence micrograph sequence of pressure-driven gated injection. (a) Fluorescing sample stream diverted into bypass channel; (b) pressure release on mobile phase stream causes retreat of latter, allowing sample stream to flow into separation channel (towards the right); (c) detachment of sample plug when mobile phase stream flow is re-established after pressure perturbation.

Please cite this article in press as: M. Schlund et al., Continuous sampling and analysis by on-chip liquid/solid chromatography, Sens. Actuators B: Chem. (2006), doi:10.1016/j.snb.2006.10.021 


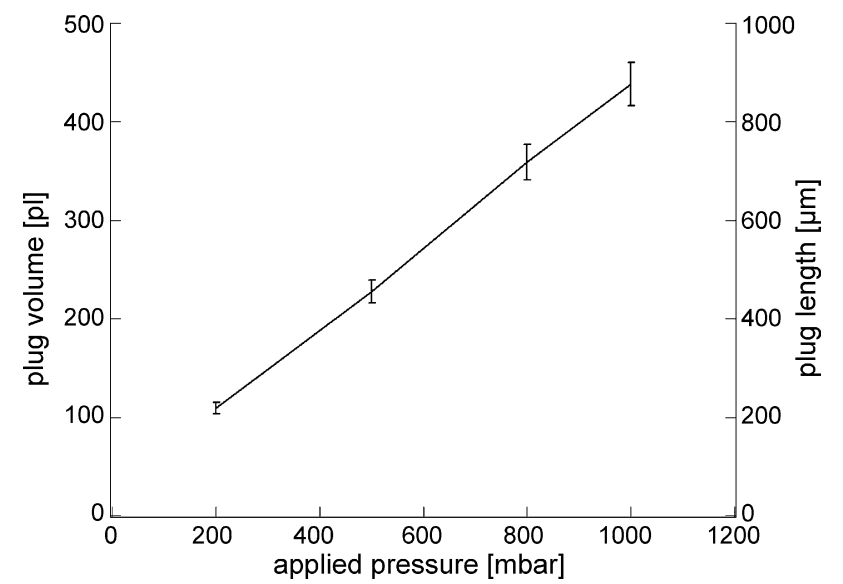

Fig. 4. Plug volume in function of the applied pressure drop (flow speed). The data are from five samples with four different injection times each. The reproducibility error is lower than $5 \%$ (error bars). Column dimensions are $L \times w \times h: 11 \mathrm{~cm} \times 50 \mu \mathrm{m} \times 10 \mu \mathrm{m}$.

Therefore, for verification of the fluorescence measurements, the peak areas of five subsequent samples with four different injection times each were analyzed (data not shown) and agreed well with the fluorescence data. Fig. 4 shows the linear trend of the plug volume versus plug injection time of the peak area analysis and confirms the observations made during fluorescence measurements. Repeated injections of analyte and its detection showed volume variations of less than $5 \%$.

To measure the influence of injection volume on peak shape, and to monitor column mass overloading, phenol was injected at a concentration of $5 \mathrm{mM}$ into a mobile phase stream consisting of $\mathrm{H}_{2} \mathrm{O} /$ acetonitrile (90:10, v/v). Peaks were recorded for various injection volumes of 200, 500, 1000 and $1500 \mathrm{~ms}$ corresponding to $70,175,350$ and $525 \mathrm{pl}$. An overlay of the single peak chromatograms recorded for each injection volume is shown in Fig. 5. Reasonably Gaussian peak shapes are evident, with increasing asymmetry due to tailing. This point is addressed in the next section. For injection times up to $1000 \mathrm{~ms}(350 \mathrm{pl})$, the

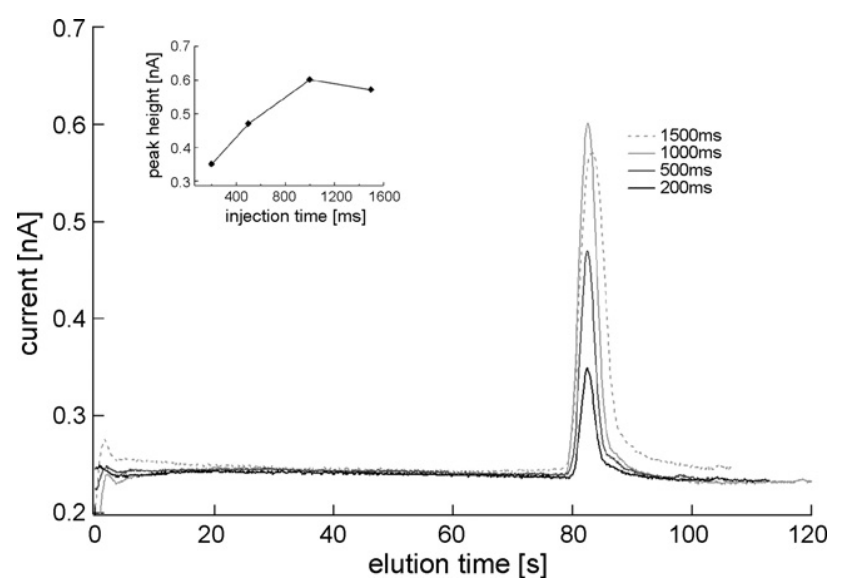

Fig. 5. Example of different injection times. Stationary phase: sol-gel $\mathrm{C}_{8}$, mobile phase: $\mathrm{H}_{2} \mathrm{O}$ and $\mathrm{ACN}(90: 10$, v/v). The pressure drop was 1000 mbar. Analyte: $5 \mathrm{mM}$ phenol. Four injections with different injection times are compared: (a) $1500 \mathrm{~ms}$; (b) $1000 \mathrm{~ms}$; (c) $500 \mathrm{~ms}$; (d) $200 \mathrm{~ms}$. For injection times up to $1000 \mathrm{~ms}(350 \mathrm{pl})$, the peak heights increased linearly. peak heights increased linearly, as illustrates the inset of Fig. 5. Peak heights appear to quickly reach a steady value at injection volumes above $350 \mathrm{pl}$ in this system, and this can be attributed purely physical zone dispersion behavior, as observed in nonchromatographic flow injection systems. In such systems, the peak maximum follows a linear dependence on sample volume up to a point, then approaches a constant value asymptotically following an exponential law [44].

The peak width increased with injection volume as expected, with greater degrees of tailing. Theoretically, peak distortion due to sample mass overload occurs when the injected sample plug occupies more than $\sqrt{N}$ theoretical plates [45], corresponding to a fraction of the total column length. In this case, the measured $N$ for the smallest peak in Fig. 5 is approximately 2200. The maximum fraction to be occupied by the plug immediately after injection is then $\sqrt{N} / N$, or $2.1 \%$ of the total column volume of $38,500 \mathrm{pl}$, which corresponds to $820 \mathrm{pl}$ or a $1560 \mathrm{~ms}$ injection. Thus, $1600 \mathrm{~ms}$ would appear to be the upper limit of experimental injection times before mass overloading would occur in this column. This is in accordance with the observation made in Fig. 5, where the $1500 \mathrm{~ms}$ injection shows a beginning tailing of the peak.

\subsection{Chromatographic separations}

The chromatographic separation tests were carried out using different phenol solutions in water/acetonitrile mobile phase. Ethanol was used for the cleaning cycles. Fig. 6 shows a typical chromatogram of four subsequent injections and also the upper limit of separation power of the microchips presented in this work. The analyte contained a mixture of $5 \mathrm{mM}$ phenol and $5 \mathrm{mM}$ 2,6-dimethylphenol. The best retention occurred in a 90:10 (v/v) water/acetonitrile mobile phase, while higher water content lead to no retention. This phenomenon is known as stationary phase breakdown in open-tubular reversed-phase chromatography. With Eq. (1), taking into account the asymme-

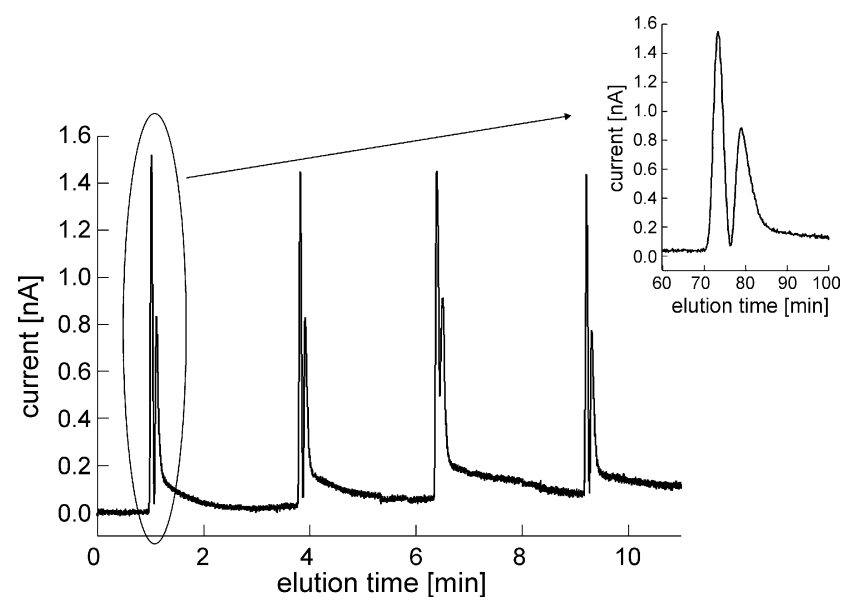

Fig. 6. Chromatogram of four subsequent injections of a two-component test solution containing $5 \mathrm{mM}$ phenol and $5 \mathrm{mM}$ 2,6-dimethylphenol in a 90:10 (v/v) $\mathrm{H}_{2} \mathrm{O}$ /acetonitrile mobile phase. The applied pressure drop is $800 \mathrm{mbar}$ and the plug volume $240 \mathrm{pl}$. Electrochemical detection with $U=1 \mathrm{~V}$. Column dimensions are $L \times w \times h: 11 \mathrm{~cm} \times 50 \mu \mathrm{m} \times 7 \mu \mathrm{m}$. 
try of the plugs with the momentum method [46] and under the same conditions as shown in Fig. 6, the best theoretical plate number $N$ obtained is approximately 4500 with a variation of less than $5 \%$ over five subsequent samples.

Number of theoretical plates:

$N=41.7 \frac{\left(t_{\mathrm{R}} / w_{0.1}\right)^{2}}{T+1.25}$

Retention factor:

$k^{\prime}=\frac{t_{\mathrm{R}}-t_{0}}{t_{0}}$

Separation factor:

$\alpha=\frac{t_{\mathrm{R}_{2}}-t_{0}}{t_{\mathrm{R}_{1}}-t_{0}}$

where $t_{\mathrm{R}}$ is the retention time (elution of retained solution), $t_{0}$ the dead time (elution time of unretained solution), $w_{0.1}$ the peak width at $10 \%$ of the peak height, $T$ the tailing factor, $k^{\prime}$ the stationary phase capacity factor (retention factor), $\alpha$ the separation factor, and $t_{\mathrm{R}_{1}}$ and $t_{\mathrm{R}_{2}}$ are the retention times of the two analytes.

A saturated uracil solution was used to determine the dead time $t_{0}$. The retention factors $k^{\prime}$ obtained were $<0.7$, while the separation factor $\alpha$ given by Eq. (3) showed typical values of around 3. For the sake of comparison, Manz et al. [6] reported a retention factor of 3.3 for their open tubular LC column on chip, while McEnery [12] showed performances below presented values.

The device presented herein is capable of doing subsequent separation, with or without washing cycles in-between. Under the chromatographic conditions of the phenol/dimethylphenol separation shown in Fig. 10, we achieve up to 20 separations/h with an injection every $3 \mathrm{~min}$. If a washing cycle in-between two subsequent separations is preferred, e.g. for changing analyte and or mobile phase, we obtain up to 10 injections/h with an injection every 6 min.

\subsection{Vitamin separations}

This paragraph shall show the very promising application of the microchip for vitamin separations. Vitamins are suitable to liquid/solid separation methods as well as to electrochemical detection.

Fig. 7 shows a typical chromatogram of Vitamins A and E separation. We can see that no baseline separation could be achieved, but the two peaks are clearly distinguishable.

The analyte contained a mixture of $1 \mathrm{mM}$ Vitamin A and $1 \mathrm{mM}$ Vitamin E. The mobile phase was again 90:10 (v/v) water/acetonitrile mobile phase. Both Vitamins $\mathrm{A}$ and $\mathrm{E}$ are fat soluble and were prepared in pure acetonitrile. Water was then added to obtain the same composition as the mobile phase (90:10, v/v, water/acetonitrile). Prior to injection into the microchip, both mixtures have been filtered in a $0.2 \mu \mathrm{m}$ pore-size Teflon ${ }^{\circledR}$ filter in order to remove agglomerates and impurities.

The separation in Fig. 7 yields a separation factor $\alpha$ of 1.3, still with respect to the unretained uracil solution. This separation shows again the limit of the actual separation power of

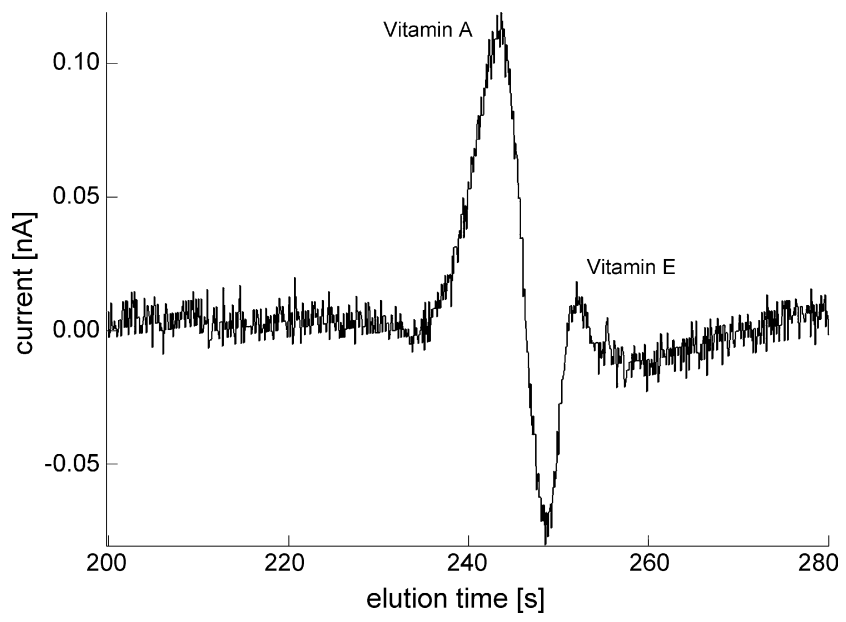

Fig. 7. Chromatogram of a vitamin solution containing $1 \mathrm{mM}$ Vitamin $\mathrm{A}$ and $1 \mathrm{mM}$ Vitamin $\mathrm{E}$ in a 90:10 (v/v) $\mathrm{H}_{2} \mathrm{O}$ /acetonitrile mobile phase. The applied pressure drop is $300 \mathrm{mbar}$ and the plug volume $100 \mathrm{pl}$. Electrochemical detection with $U=1 \mathrm{~V}$. Column dimensions are $L \times w \times h: 11 \mathrm{~cm} \times 50 \mu \mathrm{m} \times 7 \mu \mathrm{m}$.

the microchip, which is essentially due to the relatively poor stationary phase coating of the separation column.

\section{Summary and conclusion}

We report a novel design for continuous sampling and injection in chip-based pressure-driven liquid/solid chromatography. This approach operates on the principle of gated-injection by means of a novel microchannel configuration and flow control method to achieve such behavior using pressure drive. By capitalizing on the advantages of open tubular capillary LC, rational column design allows operation at low pressures to achieve optimal flow rates in short micromachined channels $(<10 \mathrm{~cm})$ that can approximately produce 4500 theoretical plates. An opentubular separation column with $\mathrm{C}_{8}$ stationary phase coating and a pressure-driven gated injection method has been demonstrated, allowing multiple analyses with rapid cycle times of a few minutes only. Sample injections down to $100 \mathrm{pl}$ with volume variations of less than $5 \%$ were achieved. It was also shown that the retention times were reproducible, indicating that the overall fluid handling system reported herein for continuous flow sampling exhibits a great deal of robustness.

A two-component phenolic test mixture has been separated with baseline resolution. The capacity factors $k^{\prime}$ of the asformed stationary phases are relatively low, although the separation factor of 3 showed sufficient selectivity for the phenol/dimethylphenol separation. This can be remedied by increasing the phase ratio of the column, making thicker and perhaps more porous sol-gel layers.

A two component vitamin mixture was separated with partial resolution. The phase ratio of the column was insufficient to fully resolve the two peaks, although the two peaks were clearly visible. However, this application shows the future potential of the present system for various applications with electrochemically detectable analytes.

Future work will indeed focus on the stationary phase development with the aim of finding reversed phase wall coatings with 
significantly increased retentive capacity factors $k^{\prime}$ and optimizing separation channel dimensions for rapid baseline separations to enhance low pressure operation without a particle packing.

\section{Acknowledgements}

The authors would like to thank Prof. em. Ervin Kovats for numerous fruitful discussions about chromatographic separations, the staff from CMI-EPFL for their support during clean-room manufacturing of the chip and Pascal Zbinden for the outstanding work done on the chip holder setup.

\section{References}

[1] J. Khandurina, A. Guttman, Microscale separation and analysis, Curr. Opin. Chem. Biol. 7 (2003) 595-602.

[2] L.J. Kricka, Miniaturization of analytical systems, Clin. Chem. 44 (1998) 2008-2014.

[3] A.W. Moore, S.C. Jacobson, J.M. Ramsey, Microchip separations of neutral species via micellar electrokinetic capillary chromatography, Anal. Chem. 67 (1995) 4184-4189.

[4] W.-C. Sung, H. Makamba, S.-H. Chen, Chip-based microfluidic devices coupled with electrospray ionization-mass spectrometry, Electrophoresis 26 (2005) 1783-1791.

[5] E. Verpoorte, Microfluidic chips for clinical and forensic analysis, Electrophoresis 23 (2002) 677-712.

[6] A. Manz, Y. Miyahara, J. Miura, Y. Watanabe, H. Miyagi, K. Sato, Design of an open-tubular column liquid chromatograph using silicon chip technology, Sens. Actuators B, Chem. 1 (1990) 249-255.

[7] G. Ocvirk, E. Verpoorte, A. Manz, M. Grasserbauer, H.M. Widmer, High performance liquid chromatography partially integrated onto a silicon chip, Anal. Methods Instrum. 2 (1995) 74-82.

[8] D. Figeys, S.P. Gygi, G. McKinnon, R. Aebersold, An integrated microfluidics-tandem mass spectrometry system for automated protein analysis, Anal. Chem. 70 (1998) 3728-3734.

[9] B. He, N. Tait, F. Regnier, Fabrication of nanocolumns for liquid chromatography, Anal. Chem. 70 (1998) 3790-3797.

[10] M. McEnery, J.D. Glennon, J. Alderman, S.C. O'Mathuna, Fabrication of a miniaturized liquid chromatography separation channel on silicon, J. Capillary Electrophor. Microchip Technol. 6 (1999) 33-36.

[11] H. Bjorkman, C. Ericson, S. Hjerten, K. Hjort, Diamond microchip capillary chromatography of proteins, MicroTAS 2000 (2000) 187-190.

[12] M. McEnery, A. Tan, J.D. Glennon, J. Alderman, J. Patterson, S.C. O'Mathuna, Liquid chromatography on-chip: progression towards a mtotal analysis system, Analyst 125 (2000) 25-27.

[13] H. Bjorkman, C. Ericson, S. Hjerten, K. Hjort, Diamond microchips for fast chromatography of proteins, Sens. Actuators B, Chem. B79 (2001) 71-77.

[14] N. Goedecke, A. Manz, Towards evaporation-driven HPLC on a chip: an alternative transport process for microanalysis systems, MicroTAS 2001 (2001) 375-376

[15] A.P. O'Neill, P. O'Brien, J. Alderman, D. Hoffman, M. McEnery, J. Murrihy, J.D. Glennon, On-chip definition of picolitre sample injection plugs for miniaturised liquid chromatography, J. Chromatogr. A 924 (2001) 259-263.

[16] G. Desmet, N. Vervoort, D. Clicq, A. Huau, P. Gzil, G.V. Baron, Shear-flowbased chromatographic separations as an alternative to pressure-driven liquid chromatography, J. Chromatogr. A 948 (2002) 19-34.

[17] D. Clicq, N. Vervoort, G.V. Baron, G. Desmet, Shear-driven flow LC. Dispensing with pumps and voltage supplies for increased speed and resolution, LC-GC Europe 17 (2004) 278-280, 282-284, 286, 288, 290.

[18] X. Yang, G. Jenkins, J. Franzke, A. Manz, Shear-driven pumping and Fourier transform detection for on chip circular chromatography applications, Lab on a Chip 5 (2005) 764-771.

[19] H. Yin, K. Killeen, R. Brennen, D. Sobek, M. Werlich, T.v.d. Goor, Microfluidic chip for peptide analysis with an integrated HPLC Column, sample enrichment column, and nanoelectrospray tip, Anal. Chem. 77 (2005) 527-533.

[20] W. Ro Kyung, J. Liu, R. Knapp Daniel, Plastic microchip liquid chromatography-matrix-assisted laser desorption/ionization mass spectrometry using monolithic columns, J. Chromatogr. A 1111 (2006) $40-47$.

[21] A. De Mello, On-chip chromatography: the last 20 years, Lab on a Chip 2 (2002) $48 \mathrm{~N}-54 \mathrm{~N}$

[22] H.J.L.X. Bai, J.L. Rossier, F. Reymond, H. Schafer, M. Wossner, H.H. Girault, Pressure-pinched injection of nanolitre volumes in planar microanalytical devices, Lab on a Chip 2002 (2002) 45-49.

[23] D. Sinton, L. Ren, D. Li, A dynamic loading method for controlling onchip microfluidic sample injection, J. Colloid Interface Sci. 266 (2003) 448-456.

[24] P.G. Vahey, S.H. Park, B.J. Marquardt, Y. Xia, L.W. Burgess, R.E. Synovec, Development of a positive pressure driven micro-fabricated liquid chromatographic analyzer through rapid-prototyping with poly(dimethylsiloxane); optimizing chromatographic efficiency with sub-nanoliter injections, Talanta 51 (2000) 1205-1212.

[25] D. Solignac, M.A.M. Gijs, Pressure pulse injection: a powerful alternative to electrokinetic sample loading in electrophoresis microchips, Anal. Chem. 75 (2003) 1652-1657.

[26] S.C. Jacobson, L.B. Koutny, R. Hergenroeder, A.W. Moore, J.M. Ramsey, Microchip capillary electrophoresis with an integrated postcolumn reactor, Anal. Chem. 66 (1994) 3472-3476.

[27] S.C. Jacobson, S.V. Ermakov, J.M. Ramsey, Minimizing the number of voltage sources and fluid reservoirs for electrokinetic valving in microfluidic devices, Anal. Chem. 71 (1999) 3273-3276.

[28] R.D. Rocklin, R.S. Ramsey, J.M. Ramsey, A microfabricated fluidic device for performing two-dimensional liquid-phase separations, Anal. Chem. 72 (2000) 5244-5249.

[29] J. Wang, W. Siangproh, S. Thongngamdee, O. Chailapakul, Continuous monitoring with microfabricated capillary electrophoresis chip devices, Analyst 130 (2005) 1390-1394.

[30] S. Büttgenbach, R. Wilke, A capillary electrophoresis chip with hydrodynamic sample injection for measurements from a continuous sample flow, Anal. Bioanal. Chem. 383 (2005) 733-737.

[31] S.-H. Chen, Y.-H. Lin, L.-Y. Wang, C.-C. Lin, G.-B. Lee, Flow-through sampling for electrophoresis-based microchips and their applications for protein analysis, Anal. Chem. 74 (2002) 5146-5153.

[32] Y.-H. Lin, G.-B. Lee, C.-W. Li, G.-R. Huang, S.-H. Chen, Flow-through sampling for electrophoresis-based microfluidic chips using hydrodynamic pumping, J. Chromatogr. A 937 (2001) 115-125.

[33] S. Lunte, B. Huynh, P. Nandi, R.S. Martin, B. Fogarty, On-line Microdialysis-Microchip Electrophoresis, Abstracts 40th Midwest Regional Meeting of the American Chemical Society, Joplin, MO, United States, October 26-29, 2005, LIN05-087.

[34] A. Berthold, L. Nicola, P.M. Sarro, M.J. Vellekoop, Glass-to-glass anodic bonding with standard IC technology thin films as intermediate layers, Sens. Actuators A, Phys. 82 (2000) 224-228.

[35] D.-J. Lee, Y.-H. Lee, J. Jang, B.-K. Ju, Glass-to-glass electrostatic bonding with intermediate amorphous silicon film for vacuum packaging of microelectronics and its application, Sens. Actuators A, Phys. 89 (2001) $43-48$.

[36] S. Constantin, R. Freitag, Preparation of stationary phases for open-tubular capillary electrochromatography using the sol-gel method, J. Chromatogr. A 887 (2000) 253-263.

[37] S. Constantin, R. Freitag, D. Solignac, A. Sayah, M.A.M. Gijs, Utilization of the sol-gel technique for the development of novel stationary phases for capillary electrochromatography on a chip, Sens. Actuators B, Chem. 78 (2001) 267-272.

[38] J.C. Giddings, J.P. Chang, M.N. Myers, J.M. Davis, K.D. Caldwell, Capillary liquid chromatography in field flow fractionation-type channels, J. Chromatogr. 255 (1983) 359-379.

[39] H. Poppe, Mass transfer in rectangular chromatographic channels, J. Chromatogr. A 948 (2002) 3-17.

[40] S.K. Griffiths, R.H. Nilson, Low-dispersion turns and junctions for microchannel systems, Anal. Chem. 73 (2001) 272-278. 
[41] S.K. Griffiths, R.H. Nilson, Design and analysis of folded channels for chip-based separations, Anal. Chem. 74 (2002) 2960-2967.

[42] E.F. Hasselbrink, T.J. Sheppod, J.E. Rehm, High-pressure microfluidic control in lab-on-a-chip devices using mobile polymer monoliths, Anal. Chem. 74 (2002) 4913-4918.

[43] D.J. Harrison, A. Manz, Z. Fan, H. Luedi, H.M. Widmer, Capillary electrophoresis and sample injection systems integrated on a planar glass chip, Anal. Chem. 64 (1992) 1926-1932.

[44] J. Ruzicka, E.H. Hansen, Flow Injection Analysis, J. Wiley and Sons, New York, 1981.

[45] H. Poppe, J.C. Kraak, Mass loadability of chromatographic columns, J. Chromatogr. 255 (1983) 395-414.

[46] V.R. Meyer, Practical High-Performance Liquid Chromatography, fourth ed., 2004.

\section{Biographies}

Mario Schlund was born in Zürich, Switzerland, in 1976. He received a master degree in microengineering with a specialization in "Micro-and Nano Systems" from the Swiss Federal Institute of Technology, Lausanne (EPFL) in 2002. He then joined the Microsystems Laboratory (LMIS) of EPFL and worked on chip based pressure driven chromatography in collaboration with Crystal Vision Microsystems. Since the fall of 2003, he has been pursuing a $\mathrm{PhD}$ in the field of chip-based pressure-driven chromatography.

Scott Gilbert received his $\mathrm{PhD}$ degree in physical chemistry from the University of California, Santa Barbara, in 1993. He subsequently accepted a postdoctoral position at the Swiss Federal Institute of Technology, Lausanne (EPFL), Institute of Physical Chemistry, for work on nanostructured liquid junction photovoltaic devices. Afterwards, he moved to the Department of Physics at EPFL where his interests expanded to explore novel methods for ultra-thin metallic film and magnetic nanowire electrodeposition and electrical contacting methods. He then joined the Microsystems Laboratory (LMIS) as project leader for the development of new technologies for miniature inductive devices, electrochemical micromachining, and branching out to microfluidics. After having worked on lab-on-chip diagnostic device development, he founded Crystal
Vision Microsystems Ltd in late 2000 to provide new solutions to the chemical, pharmaceutical and biotechnology industries via MEMS lab-on-chip technology. He is now based in Seattle, USA, where he is advancing development of lab-on-chip liquid chromatographic systems for chemical and biochemical process monitoring, in collaboration with the Center for Process Analytical Chemistry (CPAC) at the University of Washington.

Silvan Schnydrig was born 1977 in Visp, Switzerland. He received a master degree in microengineering with specialization in "Micro-and Nano Systems" in 2005 . His master thesis work was dedicated to the experimental study of the device presented in this article.

Philippe Renaud (born in 1958) received his diploma in physics from the University of Neuchatel, Switzerland (1983), and his PhD degree from the University of Lausanne, Switzerland (1988). His thesis work was dedicated to the theoretical and experimental study of magnetoelastic effects. Post-doctoral researcher at University of California, Berkeley, USA (1988-1989), to develop scanning tunneling microscopes for low temperature applications. Subseqently, he was awarded a postdoctoral postition at the IBM Zürich Research Laboratory from 1990 to 1991, where he performed measurements of the local STM induced luminescence III-V semiconductor heterostructures, studying characteristics of light emitted after injection of spin polarized electrons. In 1992, he joined the Sensors and Actuators group of the Swiss Center for Electronics and Microtechnology (CSEM), Neuchatel, Switzerland. He was involved in the design and the technology of mechanical microsensors and of micro-mirrors for optical switching. In 1993, assistant professor at the Swiss Federal Institute of Technology (EPFL). Until end of 1994, he remained part-time collaborator of CSEM. In summer 1996, visiting professor at the Tohoku University, Japan. In 1997, he was appointed as full professor at EPFL. His research interests are: microsystem design, microfabrication technologies for MEMS applications and microfluidics, BioMEMS applications. His duties extend to the directorship of EPFL's Center of MicroNanoTechnology (CMI), one of the largest state-of-the-art academic clean room facilities in the world, with processing equipment for training and scientific experimentation in microelectronic and microfabrication processes. In addition, he is chairman of the International Nanotech-Conference on Microand Nanotechnologies for Biosciences held annually in Montreux, Switzerland. 